\title{
Basal-Like Breast Cancer Defined by Five Biomarkers Has Superior Prognostic Value than Triple-Negative Phenotype
}

\author{
Maggie C.U. Cheang, ${ }^{1}$ David Voduc, ${ }^{1,3}$ Chris Bajdik, ${ }^{2}$ Samuel Leung, ${ }^{1}$ Steven McKinney, ${ }^{1}$ Stephen K. Chia, ${ }^{3}$ \\ Charles M. Perou, ${ }^{4}$ and Torsten O. Nielsen ${ }^{1}$
}

\begin{abstract}
Purpose: Basal-like breast cancer is associated with high grade, poor prognosis, and younger patient age. Clinically, a triple-negative phenotype definition [estrogen receptor, progesterone receptor, and human epidermal growth factor receptor (HER) -2, all negative] is commonly used to identify such cases. EGFR and cytokeratin $5 / 6$ are readily available positive markers of basallike breast cancer applicable to standard pathology specimens. This study directly compares the prognostic significance between three- and five-biomarker surrogate panels to define intrinsic breast cancer subtypes, using a large clinically annotated series of breast tumors.

Experimental Design: Four thousand forty-six invasive breast cancers were assembled into tissue microarrays. All had staging, pathology, treatment, and outcome information; median follow-up was 12.5 years. Cox regression analyses and likelihood ratio tests compared the prognostic significance for breast cancer death-specific survival (BCSS) of the two immunohistochemical panels.

Results: Among 3,744 interpretable cases, $17 \%$ were basal using the triple-negative definition (10year BCSS, $67 \%$ ) and $9 \%$ were basal using the five-marker method (10-year BCSS, 62\%). Likelihood ratio tests of multivariable Cox models including standard clinical variables show that the fivemarker panel is significantly more prognostic than the three-marker panel. The poor prognosis of triple-negative phenotype is conferred almost entirely by those tumors positive for basal markers. Among triple-negative patients treated with adjuvant anthracycline-based chemotherapy, the additional positive basal markers identified a cohort of patients with significantly worse outcome.

Conclusions: The expanded surrogate immunopanel of estrogen receptor, progesterone receptor, human HER-2, EGFR, and cytokeratin $5 / 6$ provides a more specific definition of basal-like breast cancer that better predicts breast cancer survival.
\end{abstract}

Breast cancer is a heterogeneous disease, and by gene expression profiling has been shown to be classifiable into five major biologically distinct intrinsic subtypes: luminal A, luminal B, human epidermal growth factor receptor-2 (HER2) overexpressing, basal-like, and normal-like (1-3). These

Authors' Affiliations: 'Genetic Pathology Evaluation Centre, Vancouver Coastal Health Research Institute, British Columbia Cancer Agency, and University of British Columbia; ${ }^{2}$ Cancer Control Research Program, BC Cancer Agency; ${ }^{3}$ BC Cancer Agency, Vancouver, British Columbia, Canada; and ${ }^{4}$ University of North Carolina, Chapel Hill, North Carolina

Received 7/5/07; revised 9/27/07; accepted 11/12/07.

Grant support: National Cancer Institute Strategic Partnering to Evaluate Cancer Signatures program (UO1-CA114722), National Cancer Institute Breast Specialized Programs of Research Excellence program (P50-CA58223-09A1), and a Canadian Breast Cancer Research Alliance Translational Acceleration Grant. C. Bajdik and T.O. Nielsen are scholars of the Michael Smith Foundation for Health Research. The Genetic Pathology Evaluation Centre is supported by an unrestricted educational grant from sanofi-aventis.

The costs of publication of this article were defrayed in part by the payment of page charges. This article must therefore be hereby marked advertisement in accordance with 18 U.S.C. Section 1734 solely to indicate this fact.

Note: Supplementary data for this article are available at Clinical Cancer Research Online (http://clincancerres.aacrjournals.org/).

Requests for reprints: Torsten O. Nielsen, Anatomical Pathology, JP 1401, Vancouver Hospital and Health Sciences Centre, 855 West 12th Avenue, Vancouver, BC, Canada V5Z1M9. Phone: 604-875-5555, ext. 62649; Fax: 604875-4497; E-mail: torsten@interchange.ubc.ca.

(C) 2008 American Association for Cancer Research.

doi:10.1158/1078-0432.CCR-07-1658 molecular subtypes have prognostic and predictive value: the HER2-overexpressing and basal-like breast cancers have poor outcomes, and within the estrogen receptor (ER)-positive subtypes the luminal $\mathrm{B}$ cohort has a significantly worse prognosis than luminal A. Follow-up studies have shown these subtypes to be conserved across diverse patient series and array platforms $(4,5)$, and have shown that different gene expression-based predictors are likely tracking a similar, common set of biological subtypes, with significant agreement in predicting patient outcome (6).

Among the five intrinsic subtypes, basal-like breast cancers have drawn particular attention, because they express neither ER, progesterone receptor (PR), nor HER2, and therefore would not be expected to benefit from antiestrogen hormonal therapies nor from trastuzumab (7). Cost and complexity issues have to date rendered gene expression profiling impractical as a routine hospital diagnostic tool. However, there are immunohistochemistry surrogate panels proposed that can potentially identify basal-like breast cancer, including ER-PR-HER2 - negative [the "triple-negative phenotype" (TNP); ref. 8], and negative hormone receptors and HER2 but either epidermal growth factor receptor (EGFR) or cytokeratin 5/6 (CK5/6) positive (the "five-marker method"; refs. 9, 10). The TNP is convenient because it applies standard biomarkers already routinely ordered during the clinical work-up of breast cancer biopsies; however, this approach has never been formally validated by correlating to 
are associated with younger patient age $(13,14)$. A readily available prognostic immunohistochemical surrogate, easily applied on formalin-fixed, paraffin-embedded biopsy tissues, would identify a cohort of breast cancer patients who may require more aggressive systemic therapy and who would be the most appropriate subjects for clinical trials specifically targeting the basal-like subtype.

This study aims to compare the prognostic value of two proposed surrogate immunohistochemical panels used to identity basal-like breast cancers: the TNP and the five-marker Core Basal definitions. Using a regional series of $>4,000$ primary invasive breast cancers with fully annotated clinical data, this report investigates the association of these two immunohistochemical panels with patient outcome.

\section{Materials and Methods}

Patients and tissue microarrays. The study cohort consists of 4,046 female primary invasive tumors. All patients had been referred to the British Columbia Cancer Agency between 1986 and 1992 and have staging, pathology, treatment, and follow-up information. The median follow-up time was 12.5 years. During the study era, $\sim 75 \%$ of breast cancer patients in the province were referred; the nonreferred were generally elderly or treated by mastectomy without indications for adjuvant systemic therapy (15). In British Columbia, most patients were treated according to provincial guidelines developed and disseminated by the British Columbia Cancer Agency (15). These guidelines were based on age, tumor size, lymphovascular invasion, nodal status, and ER levels determined by dextran charcoal ligandbinding assay (16). High risk was defined as node positive, or if node negative, presence of lymphovascular invasion, or tumor $>2 \mathrm{~cm}$ and ER negative $(<10 \mathrm{fmol} / \mathrm{mg})$. Patients considered as clinical "low risk" at the time of diagnosis during the study era were not given any adjuvant systemic therapy. Table 1 summarizes the tumor characteristics and treatment regimens of the breast cancer patients in this retrospective study, most of which have been previously presented (17). The Vancouver Hospital ER laboratory retained single archival blocks from each patient. Slides from these blocks were stained with $\mathrm{H} \& \mathrm{E}$ and reviewed by two pathologists to identify areas of invasive breast carcinoma. Tissue microarrays were constructed as previously described (17). A total of 17 tissue array blocks were built. This study was approved by the Clinical Research Ethics Board of the University of British Columbia and the British Columbia Cancer Agency.

Immunohistochemistry and fluorescent in situ hybridization. Immumunohistochemistry for ER, PR, HER2, EGFR, and CK5/6 was done on each set of 17 formalin-fixed, paraffin-embedded tissue slides using the standard streptavidin-biotin complex method with 3,3'-diaminobenzidine chromogen. ER (Clone SP1, LabVision) antibody was used at 1:250 dilution with a 8 -min microwave antigen retrieval in a $10 \mathrm{mmol} / \mathrm{L}(\mathrm{pH}$ 6.0) citrate buffer. Ready-to-use PR (Clone 1E2, Ventana) antibody was used following the Ventana automated stainer standard CC1 protocol. EGFR staining was done using the EGFR pharmDx kit (DAKO) with an enzymatic antigen retrieval by proteinase $\mathrm{K}$ for $5 \mathrm{~min}$. CK5/6 (Clone D5/ 16B4, Zymed Laboratories) antibody was used following the Ventana automated stainer mild CC1 protocol with 1:100 dilution. HER2 staining was done using HER2 (Clone SP3, LabVision) antibody at 1:100 dilution with heat-induced antigen retrieval using $0.05 \mathrm{~mol} / \mathrm{L}$ Tris buffer $(\mathrm{pH}$ 10.0) heated to $95^{\circ} \mathrm{C}$ in a steamer for $30 \mathrm{~min}$. For HER2 fluorescent in situ hybridization assay, slides were hybridized with probes to LSI Her-2/neu and CEP 17 with the PathVysion HER-2 DNA Probe Kit (Abbott Molecular, Inc.) according to the manufacturer's instructions, with modifications to the pretreatment and hybridization as previously described (18). Slides were counterstained with 4',6-diamidino-2-phenylindole and visualized on a Zeiss Axioplan epifluorescent microscope. Automated analysis of fluorescent in situ hybridization signals was done using a Metafer automated image acquisition and analysis system (Metasystems). The average copy number for each probe was determined and the amplification ratio was calculated as a ratio between the average copy per cell for HER2 and the average copy number for centromere 17. Biomarker expression from immunohistochemical assays were scored by two pathologists, blinded to the clinicopathologic characteristics and outcome of the breast tumors, using previously established and published criteria developed on other breast cancer cohorts. ER (17) and PR stains were considered positive if immunostaining was seen in $>1 \%$ of tumor nuclei. EGFR and CK5/ 6 stains were considered positive if any (weak or strong) cytoplasmic and/or membranous invasive carcinoma cell staining was observed (9). For HER2 status, tumors were considered positive if scored as $3+$ according to HercepTest criteria, and fluorescent in situ hybridization with amplification ratio $\geq 2.0$ was used to segregate immunohistochemically equivocal $(2+)$ results (19). All the stained tissue microarrays are digitally scanned and available for public access (username, basal4000; password, corebasal). ${ }^{5}$

Definition of breast cancer biological subtypes by immunohistochemistry. The immunohistochemical surrogate (ER, PR, HER2, EGFR, and CK5/6) defining breast cancer subtypes has been previously published $(9,10)$. In this study, we use two classification schemes: the TNP and the five-biomarker method. Basal-like breast cancer is defined differently by the two schemes. Using the TNP method, basal-like is negative for all routinely tested biomarkers: ER, PR, and HER2 (ER-PRHER2-), and this surrogate definition of basal-like is referred to as TNP in this article. Using the five-biomarker method, TNP becomes divided into two groups: (a) triple-negative cases (ER-PR-HER2-), which also positively express either EGFR or CK5/6, cases that are referred to as Core Basal in this article; and $(b)$ five-marker negative phenotype (5NP), which is triple negative and furthermore expresses neither EGFR nor CK5/6. Thus, the 5NP cases represent those cases considered basallike by the TNP method but not by the Core Basal definition. Three other biological subtype definitions are common to both schemes: HER2+/ER-PR- subtype, luminal' (ER+ and/or PR+, and HER2-), and luminal/HER2+ (ER+ and/or PR+, and HER2+; Supplementary Table S1). Tumors expressing HER2 but negative for both ER and PR were defined as HER2+/ER-PR-. Tumors expressing HER2 and one of the luminal markers (ER or PR) were defined as luminal/HER2+. Luminal/ HER $2+$ is not synonymous with the luminal B expression profile subtype because only $30 \%$ to $50 \%$ of luminal B tumors express HER2. Luminal' includes all cases that expression profiling defines as luminal $A$, as well as those remaining luminal B tumors that do not express HER2. Biomarker information was considered uninterpretable in cases where the tissue core was lost during sectioning or processing, or contained $<50$ visible invasive breast carcinoma cells. Tumors missing any of ER, PR, or HER2 data are categorized as unassigned. The two classification schemes are described in Supplementary Table S1.

Statistical analysis. All statistical analyses were carried out using SPSS 14.0 (SPSS, Inc.) and R 2.4.0. ${ }^{6}$ Differences between breast cancer subtypes with regard to clinicopathologic characteristics were examined using $x^{2}$ tests. For survival analysis, breast cancer-specific survival (BCSS) was of primary interest. Survival time was calculated as the date of a woman's diagnosis of breast cancer until her date of death. Survival times were censored if the primary or underlying cause of death was not breast cancer, or if the patient was still alive on June 30, 2004 (the date when the outcome data were collected). Univariate survival curves were generated by the Kaplan-Meier method (20) and differences in survival among the breast cancer subtypes were assessed by the log-rank test (21). For multivariate analysis, we built Cox regression models (22) to estimate the adjusted hazard ratios of breast cancer subtypes with standard clinicopathologic variables: age at diagnosis, histologic grade, tumor size, lymphovascular invasion, and number of positive axillary lymph nodes as a percentage of the total number examined (23). Only

\footnotetext{
${ }^{5}$ http://www.gpecimage.ubc.ca

${ }^{6}$ http://www.r-project.org
} 
Table 2. Clinicopathologic characteristics of the 4,046 breast cancer tumors

\begin{tabular}{|c|c|c|c|}
\hline \multirow[t]{2}{*}{ Characteristic } & Luminal' & Luminal/HER2+ & HER2 + /ER-PR- \\
\hline & Total $n=2,625$ & Total $n=222$ & Total $n=258$ \\
\hline Age, y & $n$ (\% within subtype) & $n$ (\% within subtype) & $n$ (\% within subtype) \\
\hline $50-65$ & $921(35.1)$ & $78(35.1)$ & $112(43.4)$ \\
\hline$>65$ & $1,044(39.8)$ & $74(33.3)$ & $64(24.8)$ \\
\hline Grade & n (\% within subtype) & n (\% within subtype) & $n$ (\% within subtype) \\
\hline 3 & $1,082(41.2)$ & $155(69.8)$ & $196(76)$ \\
\hline Unknown & $125(4.8)$ & $7(3.2)$ & $7(2.7)$ \\
\hline Tumor size, $\mathrm{cm}$ & $n$ (\% within subtype) & $n$ (\% within subtype) & $n$ (\% within subtype) \\
\hline$\leq 2$ & $1,455(55.4)$ & $90(40.5)$ & $105(40.7)$ \\
\hline $2-5$ & $1,041(39.7)$ & $118(53.2)$ & $127(49.2)$ \\
\hline$>5$ & $106(4.0)$ & $13(5.9)$ & $20(7.8)$ \\
\hline Unknown & $23(0.9)$ & $1(0.5)$ & $6(2.3)$ \\
\hline $\begin{array}{l}\text { Percentage of } \\
\text { positive/total } \\
\text { examined axillary } \\
\text { lymph nodes }(\%)\end{array}$ & $n$ (\% within subtype) & $n$ (\% within subtype) & $n$ (\% within subtype) \\
\hline 0 & $1,412(54.8)$ & $90(40.5)$ & $109(42.2)$ \\
\hline $0-25$ & $578(22.0)$ & $46(20.7)$ & $60(23.3)$ \\
\hline$>25$ & $511(19.5)$ & $72(32.4)$ & $78(30.2)$ \\
\hline
\end{tabular}

NOTE: Subtypes are defined by the TNP and Core Basal method.

Abbreviation: LVI, lymphovascular invasion.

cases with information for all the covariates were included in the analysis. Smoothed plots of weighted Schoenfeld residuals were used to test proportional hazard assumptions (24). Separate Cox regression models were also built for the subsets of patients $(a)$ receiving no adjuvant systemic therapy, to compare the prognostic values of the two basal-like subtype definitions for studying the natural history of breast cancer, and $(b)$ receiving adjuvant chemotherapy, to estimate the additional prognostic value of EGFR and CK5/6 for defining the basallike subtype in this setting.

To test the statistical significance of the additional biomarkers (EGFR and CK5/6) for defining the basal-like subtype, a likelihood ratio test $(25,26)$ of the differences between the nested Cox regression models was used. The null hypothesis was that the five-biomarker model did not describe BCSS differently than the three-biomarker model.

Bootstrap resampling analyses (27) were carried out $(10,000$ iterations) to assess the adequacy of the Cox model hazard ratio confidence intervals. In this study, bootstrapping involved randomly sampling the data with replacement and repeating the Cox regression analyses to assess the hazard ratios. We found the bootstrap confidence intervals were in close agreement with the model-based estimates, yielding no evidence that the model was overfitted to the data. The purpose of this study was to validate findings from other studies and to test a relatively small number of prespecified hypotheses; accordingly, we did not perform multiple comparisons corrections. All tests were two-sided and $P$ values $<0.05$ were considered statistically significant.

The data were assembled to provide $>80 \%$ power for testing hypotheses regarding the biomarkers in all patients combined, as well as for patient subgroups defined by the adjuvant therapies they received.

Supplementary data. Supplementary Tables S1 and S2 and Supplementary Fig. S1A and S1B are available on our publication supplemental Web site. ${ }^{7}$

\section{Results}

In this series of 4,046 tumors, the percentage of positive expression among interpretable cases is $69.5 \%(2,791$ of 4,015$)$ for ER, $13.0 \%$ (504 of 3,865) for HER2, 51.2\% (1,846 of 3,605) for PR, $13.3 \%$ (462 of 3,478) for EGFR, and 8.4\% (287 of 3,400 ) for CK5/6. A total of 3,744 tumors with enough information for unambiguous immunohistochemical surrogate classification were defined into breast cancer biological subtypes according to the TNP and the Core Basal classification schemes, respectively. There were no statistically significant survival differences between the 302 unassigned tumors and the 3,744 classifiable tumors (log-rank $P=0.179$ ). Among the cases with complete data for assignment by both schemes, $17 \%$ (639 of 3,744) were defined as basal-like breast cancers by the triple-negative definition, whereas $9.0 \%$ (336 of 3,744) were basal-like by the Core Basal definition (with the other 303 classified as $5 \mathrm{NP}$ ).

Clinicopathologic characteristics of breast cancer subtypes. The tumor characteristics of each breast cancer subtype are summarized in Table 2. With either classification scheme, the major breast cancer subtypes differ significantly by age, grade, tumor size, lymphovascular invasion, and percentage of positive over total dissected axillary lymph nodes. For both the TNP and the Core Basal classification, basal-like breast cancer is

\footnotetext{
${ }^{7}$ http://www.gpec.ubc.ca/index.php?content=papers/basal4000.php
} 
Table 2. Clinicopathologic characteristics of the 4,046 breast cancer tumors (Cont'd)

\begin{tabular}{|c|c|c|c|}
\hline \multirow{2}{*}{$\begin{array}{l}\text { TNP method } \\
\text { Basal (TNP) }\end{array}$} & \multicolumn{2}{|c|}{ Core Basal method } & \multirow[b]{2}{*}{ Unassigned } \\
\hline & Basal (Core Basal) & 5NP & \\
\hline Total $n=639$ & Total $n=336$ & Total $n=303$ & Total $n=302$ \\
\hline $115(18.0)$ & $71(21.1)$ & $44(14.5)$ & $23(7.6)$ \\
\hline $149(23.3)$ & 79 (23.5) & $70(23.1)$ & $48(15.9)$ \\
\hline $209(32.7)$ & $109(32.4)$ & $100(33.0)$ & $115(38.1)$ \\
\hline $12(1.9)$ & $1(.3)$ & $11(3.6)$ & $19(6.3)$ \\
\hline $115(18)$ & $35(10.4)$ & $80(26.4)$ & $114(37.7)$ \\
\hline $488(76.4)$ & $293(87.2)$ & $195(64.4)$ & $148(49)$ \\
\hline $24(3.8)$ & $7(2.1)$ & $17(5.6)$ & $21(7)$ \\
\hline$n$ (\% within subtype) & $n$ (\% within subtype) & $n$ (\% within subtype) & $n$ (\% within subtype) \\
\hline $284(44.4)$ & $152(45.2)$ & $132(43.6)$ & $159(52.6)$ \\
\hline $300(46.9)$ & $155(46.1)$ & $145(47.9)$ & $111(36.8)$ \\
\hline $351(54.9)$ & $185(55.1)$ & $166(54.8)$ & $166(55)$ \\
\hline $29(4.5)$ & $16(4.8)$ & $13(4.3)$ & $15(5)$ \\
\hline$n$ (\% within subtype) & $n$ (\% within subtype) & $n$ (\% within subtype) & $n$ (\% within subtype) \\
\hline $378(59.2)$ & $204(60.7)$ & $174(57.4)$ & $172(57.0)$ \\
\hline $127(19.9)$ & $66(19.6)$ & $61(20.1)$ & $65(21.5)$ \\
\hline $112(17.5)$ & $55(16.4)$ & $57(18.8)$ & $52(17.2)$ \\
\hline
\end{tabular}

associated with younger patient age, lower rates of lymphovascular invasion, and with the lowest percentage of positive axillary lymph node involvement. However, the two schemes do differ, with an even larger fraction of Core Basal cases being high grade $(87 \%$ grade 3$)$, and $<40$ years old $(18.8 \%)$ versus $64.4 \%$ of $5 \mathrm{NP}$ cases that were grade 3 , and $10.2 \%<40$ years. This suggests that the Core Basal classification is identifying a subset of particularly high-risk patients.

Breast cancer-specific survival by immunohistochemical subtype. The breast cancer subtypes as defined by the surrogate immunopanels differ significantly in predicting breast cancerspecific survival (Fig. 1). The HER2+/ER-PR- subtype has the worst breast cancer survival among the four subtypes. Comparing the survival probabilities between basal-like definitions, the 10-year breast cancer-specific survival for TNP is 67\% [95\% confidence interval (95\% CI), 63-70; Fig. 1A], but when the basal-like subtype is segregated into Core Basal and 5NP by adding EGFR and CK5/6 immunostaining information, the Core Basal group has significantly worse outcome, with an absolute 10\% lower 10-year BCSS than 5NP (Fig. 1B).

The breast cancer subtypes maintain significant independent prognostic value to predict breast cancer death in the Cox model, including the established prognostic factors (Table 3). Compared with luminal', which represents the most common subtype of breast cancers, TNP has a hazard ratio of 1.39 (95\% C.I. 1.17-1.66) for breast cancer-specific death. On the other hand, the Core Basal group has 1.62 times greater risk for breast cancer-specific death (95\% CI, 1.31-2.00), whereas the 5NP group does not have statistically or clinically significant prognostic value (HR, 1.16; 95\% CI, 0.91-1.49; Table 3).

The likelihood ratio test between the two Cox models is significant $(P=0.0273$; Table 3$)$.
Because the hazard ratio between luminal' and 5NP is not proportional across time, Cox regression analysis may overestimate or underestimate this significance. We ran multivariate analyses in these TNP tumors to test the significance of Core Basal association with outcome in this subset. Relative to 5NP, Core Basal has an estimated hazard ratio of 1.47 (95\% CI, 1.081.99) for breast cancer death (Table 4A). The likelihood ratio test is also significant. Therefore, in this cohort, relying on the three-biomarker classifier (ER, PR, and HER2) to define basallike tumors loses significant information to predict breast cancer outcome, compared with the five-marker panel incorporating EGFR and CK5/6.

Prognostic values of breast cancer subtypes within treatment subsets. Among the Core Basal patients, 179 received no adjuvant systemic therapy, 48 were treated with anthracyclinebased chemotherapy (32 with doxorubicin/cyclophosphamide and 16 with fluorouracil/doxorubicin/cyclophosphamide), and 55 were treated with nonanthracycline-based chemotherapy (cyclophosphamide, methotrexate, and fluorouracil). Among the 5NP patients, 141 received no adjuvant systemic therapy, 58 received anthracycline-based chemotherapy (36 doxorubicin/cyclophosphamide and 22 fluorouracil/doxorubicin/cyclophosphamide), and 31 received nonanthracycline-based chemotherapy. In the no adjuvant systemic therapy subset, although Core Basal and 5NP were ER-negative, predominantly grade 3 tumors, most had neither nodal involvement nor lymphovascular invasion and half were $<2 \mathrm{~cm}$ (Supplementary Table S2).

The Core Basal patients who received no adjuvant systemic therapy (predominantly considered clinically low risk at the time) have $9 \%$ lower 10 -year BCSS than similarly treated 5NP patients (Supplementary Fig. S1A). 


\section{A}

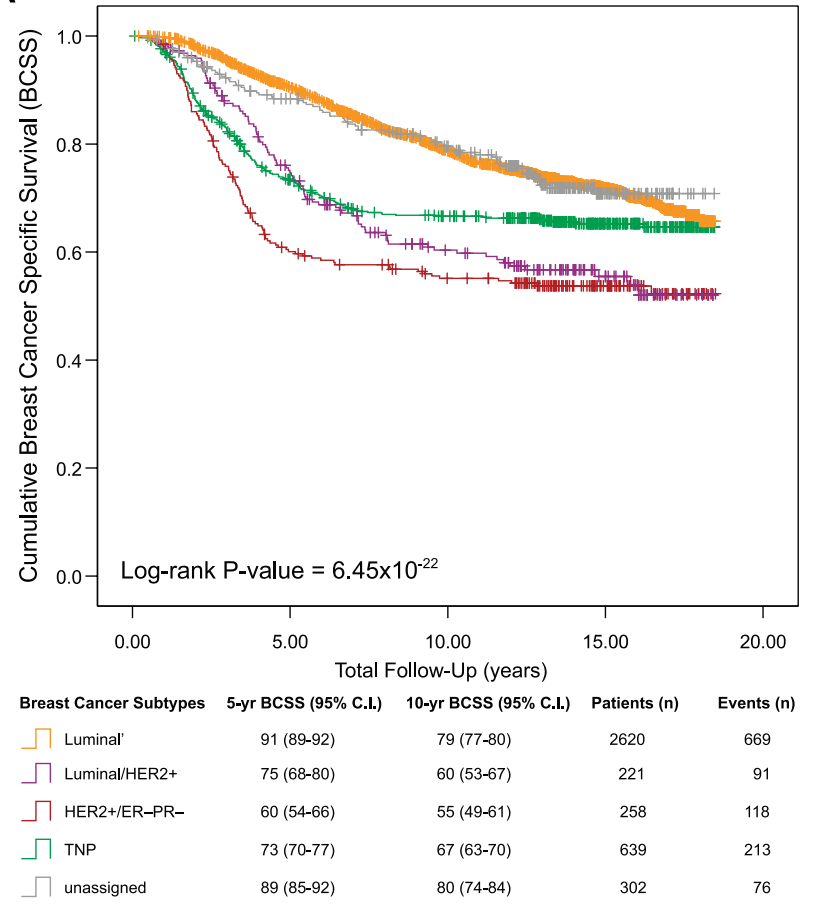

B

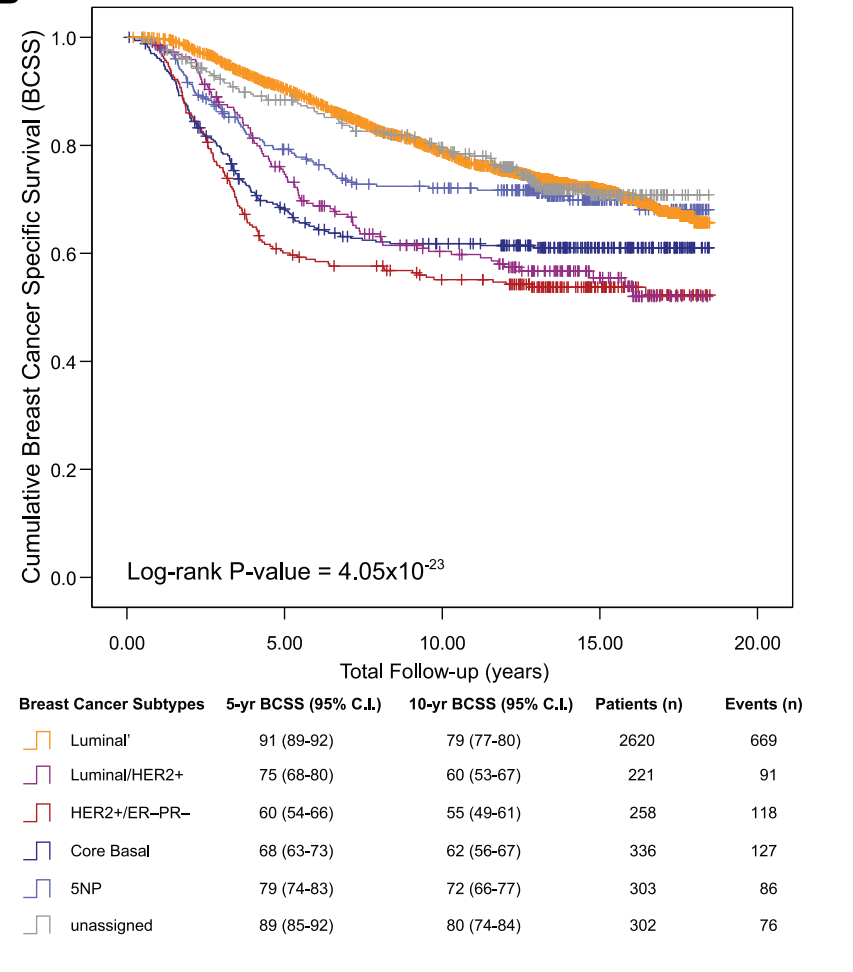

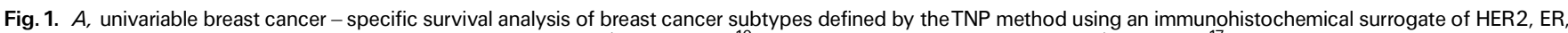

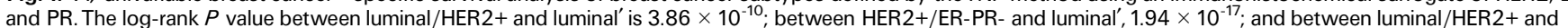

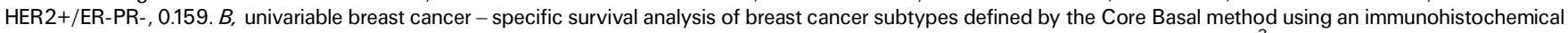

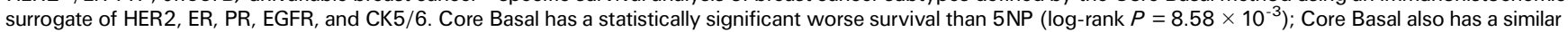
survival rate as HER2+/ER-PR-group for the first 3 y (log-rank $P=0.087$ ).

For patients treated with anthracycline-based adjuvant chemotherapy (Fig. 2), the Core Basal patients have a significant 26\% lower 10-year BCSS than equivalently treated 5 NP patients $\left(\log\right.$-rank $\left.P=1.64 \times 10^{-3}\right)$. After adjusting for age, tumor size, grade, axillary node, and lymphovascular invasion status, the Core Basal group has a significantly worse survival, with a hazard ratio of 4.26 versus the $5 \mathrm{NP}$ cohort $(95 \% \mathrm{CI}$, 2.00-9.08; Table 4B). In this patient group, Core Basal status is the most significant prognosticator in the multivariable model, ahead of nodal involvement and tumor size. The likelihood ratio test is significant $\left(P=7.41 \times 10^{-5}\right)$.

On the other hand, among tumors receiving nonanthracycline-based chemotherapy (Supplementary Fig. S1B), outcomes were relatively poor with no statistically significant survival difference between Core Basal and 5NP. The tumor characteristics between nonanthracycline (cyclophosphamide, methotrexate, and fluorouracil) versus anthracycline (doxorubicin/ cyclophosphamide or fluorouracil/doxorubicin/cyclophosphamide) treated cohorts were similar (Supplementary Table S2); tumors treated with anthracyclines were mostly diagnosed between 1990 and 1992.

\section{Discussion}

Using a large regional population-based cohort, this study compared two previously established immunohistochemical surrogate panels that define breast cancer subtypes. Prognostic implications of breast cancer molecular subtypes have been described in several reports $(1,3,8-10)$; however, to date, no studies have been this large ( $>3700$ patients) nor have assessed breast cancer-specific survival, stratified by adjuvant treatment, using the triple-negative versus five-biomarker Core Basal methods. The molecular subtyping of breast cancer is validated and shown to be an independent prognostic factor. We show that including positive markers (EGFR and cytokeratins 5/6) for the basal subtype results in a significantly better identification of a high-risk group, whose outcome more closely matches that expected by gene expression profiling $(1-3,5)$ than was achieved using a triple-negative (ER-PR-HER2-) definition. Our results from the multivariable Cox regression analyses strongly suggest that among the triple-negative cases, the poor prognosis is conferred almost entirely by the subset of tumors that are positive for EGFR or basal cytokeratins.

Basal-like breast cancer has been suggested to be definable by negative ER, PR, and HER2 immunostaining $(8,28-30)$, a TNP that can often be extracted from existing clinical records; however, this definition has never been validated with microarray data. Our results provide evidence that this definition can easily be improved upon through the use of other immunostains already commonly used in surgical pathology laboratories. The prevalence of the TNP (17\%) in our study is consistent with a recent report that assigned 281 of 1,726 cases $(16.3 \%)$ as TNP (31). This study found that among TNP cases, a basal phenotype (defined using 
CK5/6 and CK14) in a concurrent report (32) by the same group was significantly prognostic within the node-negative subset, and further suggested that triple-negative and basal definitions are associated with good response to chemotherapy (although treatments were not randomized and information on chemotherapy regimens was not given). The specific Core Basal definition used here and based on previous independent series was not presented and therefore direct comparisons are difficult to make. Another study, using 375 stage II breast tumors treated with tamoxifen but not adjuvant chemotherapy, defined 48 tumors as basal-like on the basis of cytokeratin 5 or 14 immunostaining, and reported no significant survival differences among ERnegative tumors (33). The discrepancy is likely due to limited power and the different choice of surrogate biomarkers, as cytokeratin 14 has not been found by gene expression to be a marker for basal-like tumors. In our "pure prognostic" group of patients receiving no adjuvant systemic therapy, Core Basal (10-year BCSS 70\%) and HER2+/ERPR- (10-year BCSS 59\%) subtypes are associated with significantly distinct breast cancer-specific survival in univariable Kaplan-Meier analysis (log-rank $P=0.0395)$, with an adjusted hazard ratio of 0.74 (95\% CI, 0.438-1.04). Our results support that basal-like phenotype breast tumors, having a different natural history than HER2+/ER-PR-, display a clinically distinct outcome, as well as distinct clinical features such as high grade, node-negative progression, and higher prevalence in young patients.

By adding EGFR and CK5/6 as positive markers, a significantly worse outcome group can be identified among triplenegative cases. The Core Basal definition is associated with even poorer breast cancer survival in the whole population-based cohort, and also in the anthracycline-based chemotherapy cohort, a generally high-risk group treated with similar regimens in contemporary practice. Poor outcome despite anthracycline treatment is concordant with a recent casecontrol study (47 basal cytokeratin-expressing breast cancers and 49 stage-matched but mainly ER+ controls; ref. 34). Other studies $(8,14,35)$ have reported that the basal-like subtype is a potential candidate to respond well to chemotherapy. In a neoadjuvant study, basal-like tumors (defined by TNP) had higher rates $(27 \%)$ of pathologic complete response to anthracycline-based neoadjuvant chemotherapy than luminal breast cancers (36). However, those triple-negative tumors, which did not have complete response, had the highest rate of relapse, potentially explaining the poor prognosis of basal-like tumors as a group despite aggressive chemotherapy. Our findings are compatible with a recent study done analyzing 823 patients from two clinical trials randomized to receive anthracyclines versus no adjuvant chemotherapy (37). In that study, a "true basal" group defined as HER2 negative, ER negative, and either EGFR or CK5/6 positive exhibited less benefit from anthracyclines than the group negative for all four of these markers.

One limitation of our study is that our cohort derives from a regional population base. Adjuvant! is a computer software program that predicts breast cancer outcomes based on Surveillance, Epidemiology, and End-Results data and clinical trial meta-analyses to guide treatment decisions in clinical practice (38). Almost half of our data set was used in an earlier study confirming that, in the British Columbia population, Adjuvant! predictions are comparable with observed outcomes (16), supporting extrapolation of the conclusions drawn in the present study to North American populations.

Table 3. Cox regression analysis to estimate the adjusted hazard ratios of breast cancer subtypes defined by TNP and Core Basal method, respectively, on 3,558 cases with sufficient information for all of the variables

\begin{tabular}{|c|c|c|c|}
\hline \multirow[t]{2}{*}{ Variables } & \multirow{2}{*}{$\frac{\text { Univariate analysis }}{\text { HR }(95 \% \mathrm{CI})}$} & \multicolumn{2}{|c|}{ Multivariate analysis } \\
\hline & & Nested Cox model HR (95\% CI) & Full Cox model HR ( $95 \% \mathrm{CI})$ \\
\hline \multicolumn{4}{|l|}{ Age, y } \\
\hline$>65$ vs $\leq 40$ & $0.65(0.54-0.78)$ & $0.87(0.71-1.07)$ & $0.89(0.72-1.09)$ \\
\hline \multicolumn{4}{|l|}{ Grade } \\
\hline 3 vs ( 2 and 1$)$ & $2.11(1.86-2.39)$ & $1.49(1.30-1.71)$ & $1.47(1.28-1.69)$ \\
\hline \multicolumn{4}{|l|}{ Tumor size, $\mathrm{cm}$} \\
\hline $2-5$ vs $\leq 2$ & $2.08(1.84-2.36)$ & $1.65(1.44-1.88)$ & $1.65(1.44-1.88)$ \\
\hline$>5$ vs $\leq 2$ & $3.32(2.69-4.09)$ & $1.77(1.38-2.27)$ & $1.77(1.39-2.27)$ \\
\hline \multicolumn{4}{|c|}{ Percentage of positive/dissected axillary lymph nodes } \\
\hline $0-25$ vs 0 & $1.98(1.70-2.31)$ & $1.62(1.36-1.92)$ & $1.62(1.37-1.93)$ \\
\hline$>25$ vs 0 & $3.99(3.48-4.58)$ & $2.88(2.44-3.40)$ & $2.89(2.45-3.42)$ \\
\hline \multicolumn{4}{|l|}{ Breast cancer subtype } \\
\hline TNP & $1.50(1.28-1.74)$ & $1.39(1.17-1.66)$ & \\
\hline Core Basal & $1.77(1.46-2.14)$ & & $1.62(1.31-2.00)$ \\
\hline $5 N P$ & $1.22(0.97-1.52)$ & & $1.16(0.91-1.49)$ \\
\hline
\end{tabular}

NOTE: Hazard ratios above 1.0 indicate poorer outcome. Likelihood ratio test of TNP (nested model) and Core Basal (full model) methods has a $P$ value of 0.0273 . Hazard ratios for individual clinicopathologic variables in univariable breast cancer-specific survival listed for reference. 
Table 4. Cox regression analyses on basal (TNP) tumors

A. Cox regression analysis on $\mathbf{5 7 5}$ basal (TNP) tumors with sufficient information for the clinicopathologic covariates: age, tumor size, grade, lymphovascular invasion, and percentage positive among total examined axillary lymph nodes. Likelihood ratio test, $P=0.0127, d f=1$

\begin{tabular}{lc}
\hline Variables & Hazard ratio (95\% CI) \\
\hline Age, y & \\
$40-49$ vs $\leq 40$ & $1.13(0.74-1.74)$ \\
$50-65$ vs $\leq 40$ & $1.01(0.67-1.53)$ \\
$>65$ vs $\leq 40$ & $1.11(0.71-1.74)$ \\
Grade & $1.31(0.86-1.97)$ \\
3 vs ( 2 and 1) & $1.72(1.21-2.44)$ \\
Lymphovascular invasion & \\
$\quad$ Positive vs negative & $1.68(1.22-2.32)$ \\
Tumor size, cm & $1.72(1.04-2.84)$ \\
$2-5$ vs $\leq 2$ & \\
$>5$ vs $\leq 2$ & \\
Percentage of & \\
$\quad$ positive/total number & \\
$\quad$ of dissected axillary & \\
$\quad$ lymph nodes & \\
$\quad 0-25$ vs 0 & $1.57(1.07-2.30)$ \\
$>25$ vs 0 & $2.64(1.77-3.96)$ \\
Breast cancer subtype & \\
Basal (Core Basal) vs 5NP & $1.47(1.08-1.99)$
\end{tabular}

B. Cox regression analysis on the 95 basal (TNP) tumors treated with anthracycline-based (AC or FAC) adjuvant chemotherapy with sufficient information for the clinicopathologic covariates: age, tumor size, grade, lymphovascular invasion, and percentage positive among total examined axillary lymph nodes. Likelihood ratio test, $\boldsymbol{P}=$ $7.41 \times 10^{-5}, d f=1$

\section{Variables}

Hazard ratio (95\% CI)

Age, y

$\geq 50$ vs $<50$

$1.48(0.73-3.00)$

Grade

3 vs (2 and 1 )

$0.38(0.11-1.27)$

Lymphovascular invasion

Positive vs negative

Tumor size, $\mathrm{cm}$

$>2$ vs $\leq 2$

$1.19(0.46-3.07)$

Percentage of

$2.20(0.98-4.96)$

positive/total number

of dissected axillary

lymph nodes

$0-25$ vs 0

$0.68(0.20-2.28)$

$>25$ vs 0

$5.07(1.84-14.0)$

Breast cancer subtype

Basal (Core Basal) vs 5NP

$4.26(2.00-9.08)$

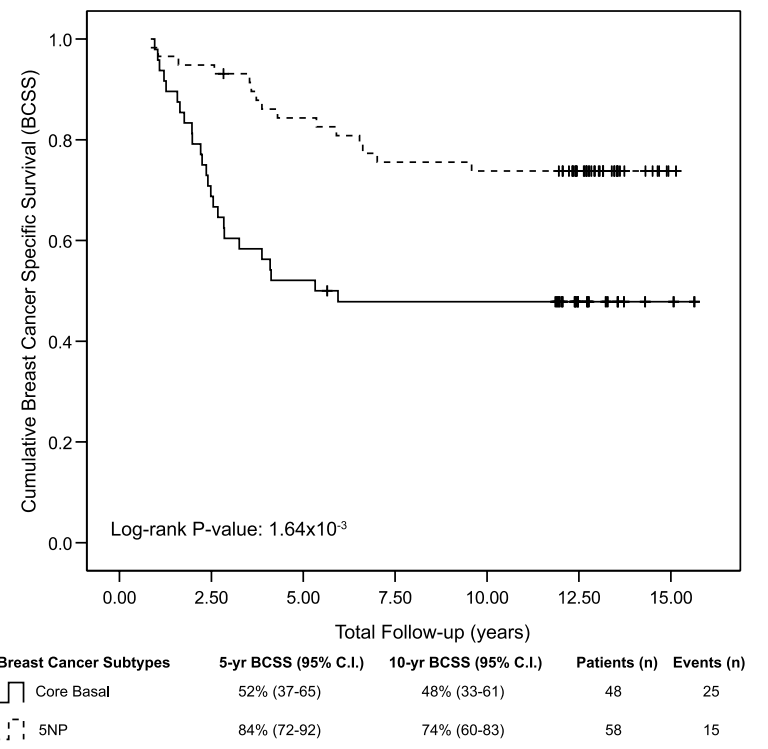

Fig. 2. Univariable breast cancer - specific analysis among 106 TNP tumors receiving anthracycline-based adjuvant chemotherapy regimen. Solid line, Core Basal cases expressing EGFR or CK5/6. Dashed line, 5NP cases expressing none of these markers.

poor outcomes of Core Basal over 5NP tumors in the adjuvant anthracycline subset) are best considered hypothesis generating. Thus, our finding that the Core Basal definition may predict response of anthracycline-based adjuvant chemotherapies needs validation. Prospective clinical trial designs are clearly needed to investigate the benefit of different chemotherapy regimens in basal-like breast cancer. Use of a triple-negative definition is attractive in the design of such studies as it does not necessitate additional biomarker information. However, a major implication of our current study is that relying on a TNP definition of basal-like breast cancer will still identify a heterogeneous group with significant differences in survival, potentially obscuring important findings.

In North American and European populations, 12\% to $20 \%$ of breast cancer patients have basal-like gene expression profiles and/or a triple-negative immunophenotype $(1,3,30$, 31 ). Our results provide strong evidence to support the use of a five-biomarker surrogate (ER, PR, HER2, EGFR, and CK5/6) to define the basal-like subtype, a finding of immediate relevance to prognostication and clinical trial design. Drawing on readily available inexpensive diagnostic tools already in clinical use, this immunopanel provides a more specific definition of this aggressive form of breast cancer for which there is a particular need to improve therapeutic options.

In British Columbia, most patients were treated according to provincial guidelines developed and disseminated by the British Columbia Cancer Agency. Associations relying on nonrandomized treatment regimens (such as the apparent

\section{References}

1. Perou CM, Sorlie T, Eisen MB, et al. Molecular portraits of human breast tumours. Nature 2000;406 747-52.

2. Sorlie T, Perou CM, Tibshirani R, et al. Gene expression patterns of breast carcinomas distinguish tumor subclasses with clinical implications. Proc Natl Acad Sci U S A 2001;98:10869-74
3. SorlieT, Tibshirani R, Parker J, et al. Repeated observation of breast tumor subtypes in independent gene expression data sets. Proc Natl Acad Sci U S A 2003 100:8418-23

4. Hu Z, Fan C, Oh DS, et al. The molecular portraits of breast tumors are conserved across microarray platforms. BMC Genomics 2006;7:96.
5. Sorlie T, Wang Y, Xiao C, et al. Distinct molecular mechanisms underlying clinically relevant subtypes of breast cancer: gene expression analyses across three different platforms. BMC Genomics 2006;7:127.

6. Fan C, Oh DS, Wessels $L$, et al. Concordance among gene-expression-based predictors for breast cancer. N Engl J Med 2006:355:560-9. 
7. Slamon DJ, Leyland-Jones B, Shak S, et al. Use of chemotherapy plus a monoclonal antibody against HER2 for metastatic breast cancer that overexpresses HER2. N Engl J Med 2001;344:783-92.

8. Haffty BG, Yang $Q$, Reiss M, et al. Locoregional relapse and distant metastasis in conservatively managed triple negative early-stage breast cancer. J Clin Oncol 2006;24:5652-7

9. Nielsen TO, Hsu FD, Jensen K, et al. Immunohisto chemical and clinical characterization of the basal-like subtype of invasive breast carcinoma. Clin Cancer Res 2004:10:5367-74

10. Carey LA, Perou CM, Livasy CA, et al. Race, breast cancer subtypes, and survival in the Carolina Breast Cancer Study. JAMA 2006;295:2492-502.

11. Abd El-Rehim DM, Pinder SE, Paish CE, et al. Expression of luminal and basal cytokeratins in human breast carcinoma. J Pathol 2004;203:661 - 71.

12. Turner N, Tutt $A$, A shworth $A$. Hallmarks of 'BRCAness' in sporadic cancers. Nat Rev Cancer 2004;4:814-9.

13. Livasy CA, Karaca G, Nanda R, et al. Phenotypic evaluation of the basal-like subtype of invasive breas carcinoma. Mod Pathol 2006;19:264-71.

14. Rodriguez-Pinilla SM, Sarrio D, Honrado $E$, et al. Prognostic significance of basal-like phenotype and fascin expression in node-negative invasive breas carcinomas. Clin Cancer Res 2006:12:1533-9.

15. Olivotto A, Coldman AJ, Hislop TG, et al. Compliance with practice guidelines for node-negative breas cancer. J Clin Oncol 1997:15:216-22.

16. Olivotto IA, Bajdik CD, Ravdin PM, et al. Populationbased validation of the prognostic model ADJU VANT! for early breast cancer. J Clin Oncol 2005;23 2716-25.

17. Cheang MC, Treaba DO, Speers $\mathrm{CH}$, et al. Immunohistochemical detection using the new rabbit monoclonal antibody SP1 of estrogen receptor in breas cancer is superior to mouse monoclonal antibody
1D5 in predicting survival. J Clin Oncol 2006;24: 5637-44.

18. Brown LA, Irving J, Parker R, et al. Amplification of EMSY, a novel oncogene on 11q13, in high grade ovarian surface epithelial carcinomas. Gynecol Oncol 2006;100:264-70.

19. Yaziji $H$, Goldstein LC, Barry TS, et al. HER-2 testing in breast cancer using parallel tissue-based methods. JAMA 2004;291:1972-7.

20. Bland JM, Altman DG. Survival probabilities (the Kaplan-Meier method). BMJ 1998;317:1572.

21. Bland JM, Altman DG. The logrank test. BMJ 2004 328:1073.

22. Cox DR, Oakes D. Analysis of survival data. London (England): Chapman \& Hall; 1984.

23. Truong PT, Berthelet E, LeeJ, Kader HA, Olivotto IA The prognostic significance of the percentage of positive/dissected axillary lymph nodes in breast cancer recurrence and survival in patients with one to three positive axillary lymph nodes. Cancer 2005;103 2006-14.

24. Grambsch $P$, Therneau T. Proportional hazards tests and diagnostics based on weighted residuals. Biometrika 1994;81:515-26.

25. Hosmer DW, Lemeshow S. Applied logistic regression. New York (NY): Wiley \& Sons; 1989

26. Tableman M, Kim JS. Survival analysis using $S$ : analysis of time-to-event data. Boca Raton: Chapman \& Hall/CRC; 2004

27. Efron B, Tibshirani RJ. An introduction to the bootstrap. Chapman \& Hall; 1994.

28. Brenton JD, Carey LA, Ahmed AA, Caldas C. Molecular classification and molecular forecasting of breast cancer: ready for clinical application? J Clin Oncol 2005:23:7350-60.

29. Jacquemier J, Padovani L, Rabayrol L, et al. Typica medullary breast carcinomas have a basal/myoepithelial phenotype. J Pathol 2005;207:260-8.
30. Bauer KR, Brown M, Cress RD, Parise CA, Caggiano $\mathrm{V}$. Descriptive analysis of estrogen receptor (ER)negative, progesterone receptor (PR)-negative, and HER2-negative invasive breast cancer, the socalled triple-negative phenotype: a population-based study from the California cancer Registry. Cancer 2007;109:1721 -8

31. Rakha EA, El-Sayed ME, Green AR, LeeAH, Robertson JF, Ellis IO. Prognostic markers in triple-negative breast cancer. Cancer 2007;109:25-32.

32. Rakha EA, El-Rehim DA, Paish C, et al. Basal phenotype identifies a poor prognostic subgroup of breast cancer of clinical importance. Eur J Cancer 2006;42:3149-56.

33. Jumppanen $M$, Gruvberger-Saal $S$, Kauraniemi $P$, et al. Basal-like phenotype is not associated with patient survival in estrogen receptor negative breast cancers. Breast Cancer Res 2007;9:R16.

34. Banerjee S, Reis-Filho JS, Ashley S, et al. Basal-like breast carcinomas: clinical outcome and response to chemotherapy. J Clin Pathol 2006;59:729-35.

35. Rouzier R, Perou CM, Symmans WF, et al. Breast cancer molecular subtypes respond differently to preoperative chemotherapy. Clin Cancer Res 2005;11: 5678-85.

36. Carey LA, Dees EC, Sawyer L, et al. The triple negative paradox: primary tumor chemosensitivity of breast cancer subtypes. Clin Cancer Res 2007;13: 2329-34.

37. Conforti R, Boulet T, Tomasic G, et al. Breast cancer molecular subclassification and estrogen receptor expression to predict efficacy of adjuvant anthracyclines-based chemotherapy: a biomarker study from two randomized trials. Ann Oncol 2007;18:1477-83.

38. Ravdin PM, Siminoff LA, Davis GJ, et al. Computer program to assist in making decisions about adjuvant therapy for women with early breast cancer. J Clin Oncol 2001;19:980-91. 